Meta

Journal des traducteurs

Translators' Journal

\title{
Les réseaux d'eau : éléments de vocabulaire
}

\section{Bruno Couture}

Volume 15, numéro 3, septembre 1970

URI : https://id.erudit.org/iderudit/002428ar

DOI : https://doi.org/10.7202/002428ar

Aller au sommaire du numéro

Éditeur(s)

Les Presses de l'Université de Montréal

ISSN

0026-0452 (imprimé)

1492-1421 (numérique)

Découvrir la revue

Citer cet article

Couture, B. (1970). Les réseaux d'eau : éléments de vocabulaire. Meta, 15(3),

173-175. https://doi.org/10.7202/002428ar d'utilisation que vous pouvez consulter en ligne.

https://apropos.erudit.org/fr/usagers/politique-dutilisation/ 


\section{LES RÉSEAUX D'EAU :}

\section{ÉLÉMENTS DE VOCABULAIRE}

On ne peut guère aborder le vocabulaire des réseaux d'eau sans établir au préalable les distinctions qui s'imposent entre les mots conduite, canalisation, conduit et tuyau. Le champ sémantique du tuyau, entre autres, ne concorde pas exactement avec celui du mot anglais pipe. Il faut donc se livrer, en l'occurrence, à une petite étude synonymique et essayer de dégager des constantes, à partir des définitions des dictionnaires et de l'usage suivi par les spécialistes ${ }^{1}$.

On constate alors que la conduite (main) est rigide, a un diamètre assez important et est généralement enterrée. La canalisation (line), appelée aussi tuyauterie (piping) et, plus rarement, tuyautage, est d'ordinaire rigide, de section plus faible et fait partie d'une installation, fixe ou montée sur un véhicule. Comme la conduite, elle est utilisée pour l'eau, le gaz, etc., quoique en termes d'électricité, canalisation correspond aussi au mot anglais raceway. Le conduit (duct), rigide, de section assez forte et souvent quadrangulaire, sert soit à évacuer des gaz de combustion ou de l'air vicié, soit à distribuer de l'air chaud ou conditionné; dans ce dernier cas, on dit aussi gaine. Signalons en outre que certains auteurs donnent à canalisation les sens de conduite et de conduit. Le tuyau, qui peut être rigide (pipe), semi-rigide (non-collapsible hose), souple ou flexible (collapsible hose), n'est qu'un des éléments à mettre bout à bout pour y faire circuler des solides, des liquides ou des gaz. Mentionnons, pour mémoire, les manches souples (flexible tubing) équipant les ventilateurs (smoke ejectors) utilisés par les pompiers pour désenfumer les locaux sinistrés, et les manches à incendie des navires. Quant à boyau, qu'on chercherait en vain dans les textes, même s'il figure encore dans tous les dictionnaires, c'est un «terme ancien désignant les tuyaux d'incendie ».

Puisque nous sommes au chapitre des distinctions, il y aurait lieu aussi, avant d'aller plus loin, de s'arrêter aux différents équivalents français du mot anglais valve, car ce terme est également capital pour la suite de notre étude. Valve peut se traduire par valve, soupape, clapet, robinet et vanne. Bien que les dictionnaires ne permettent pas de se faire une idée parfaite de ce qui distingue cette série synonymique, l'usage paraît assez bien fixé pour qu'on puisse formuler les observations sommaires suivantes.

La valve sert à retenir le fluide dans la capacité sur laquelle elle est installée (exemple : la valve d'une chambre à air), tandis que la soupape, agissant en sens inverse, permet l'évacuation d'un excédent de liquide ou de gaz (soupape de sûreté, par exemple). Le clapet (clapper) qui, avec son siège, en est l'élément principal, peut aussi s'intercaler dans une canalisation (exemple: clapet de

1. Sources bibliographiques : Grand Larousse encyclopédique, Paris, Larousse, 1960-1964; Association française de normalisation, " Matériel de secours et de lutte contre l'incendie *, Terminologie, Paris, 1969; Goyens, Manuel pratique de la lutte contre le feu et les risques associés, Paris, Larousse, p. 232; Barbier, Cardiergues et Stoskopft, Dictionnaire technique du bâtiment et des travaux publics, Paris, Eyrolles, 1964; Catalogue de la Société des fonderies de Pont-à-Mousson, Nancy, 1963; Laborderie et Sivadier, Cours élémentaire d'hydraulique à l'usage des sapeurs-pompiers, Paris, France-Sélection, 1967; Albert Odillon, Dictionnaire du feu, Paris, L'Argus, 1951; Beltramelli, Précis de prévention, Paris, FranceSélection; Revue technique du feu, $\mathrm{n}^{\mathrm{0}} 62$ (1966), p. 37; Faure et Beltramelli, le Felt, Paris, Presses Universitaires de France, 1962. 
retenue). Le robinet (valve, cock, tap, faucet) qui, seul, exige une intervention extérieure, peut être, selon l'axe de déplacement de son obturateur, à soupape ou à vanne (gate valve). Le mot vanne sert aussi à désigner les robinets de grande dimension. On voit donc qu'il est incorrect de rendre automatiquement le mot anglais valve par soupape.

Toujours dans le domaine de la robinetterie, il convient de mentionner que la fermeture trop rapide d'un robinet ou d'une vanne occasionne le coup de bélier (water hammer), qu'il ne faut pas confondre avec le marteau d'eau (également water hammer) : " tube de verre cylindrique, partiellement rempli d'eau et vide d'air ». Ajoutons que l'«espace libre, maçonné, qu'on ménage, sous terre, autour d'un robinet, afin de pouvoir le manœuvrer », se nomme tabernacle (valve pit) honni soit qui mal y pense! - et que la hub gate ou hub valve est une vanne commandée par clef à béquille.

Comme leur nom l'indique, les réseaux d'adduction et de distribution (waterworks systems), plus communément appelés réseaux d'eau (water systems), comprennent des conduites alimentaires, d'amenée ou d'adduction (supply mains), et des conduites de distribution (feeders). Un réseau dont les conduites reçoivent l'eau de deux côtés (circulating mains) est dit maillé (gridiron) ou bouclé (loop system), et ramifié s'il est constitué de conduites en impasse (dead-end mains). Étant donné l'entartrement plus ou moins rapide des conduites, leur diamètre doit être largement calculé (of generous size), de façon à réduire au minimum les pertes de charge (friction loss), causées surtout par le frottement de l'eau contre les parois.

L'appareillage hydraulique piqué sur (to tap) les conduites d'eau constitue la fontainerie (hydrants). Nous ne retiendrons ici que trois appareils principaux : le poteau d'incendie, la bouche d'incendie et la borne-fontaine. Suivant leur dispositif de fermeture, les poteaux d'incendie (post hydrants) sont de deux types : à robinet-vanne (gate hydrant) ou à clapet (compression hydrant). Ils comportent en principe, installées au-dessus du sol sur une colonne ou corps tubulaire vertical (barrel), une prise principale de face (pumper outlet, steamer connection) pour l'alimentation des engins-pompes et deux prises secondaires latérales (hose outlets) pour le branchement de lances d'incendie (fire streams).

Leurs orifices d'écoulement (butts) sont munis de demi-raccords (nipples), obturés chacun par un bouchon (cap) retenu par une chaînette. Leur nomenclature comprend aussi le chapeau (bonnet), la tige de commande (operating stem), le bourrelet (flange), le conde à patin (boot, shoe) et la bride d'admission (strapping lugs). On les soumet périodiquement à des essais hydrauliques (flow tests), afin de s'assurer qu'ils peuvent fournir, seuls ou conjointement avec d'autres ressources en eau (water supplies), ce que les services de protection appellent le débit nécessaire à la lutte contre le feu (fire flow). Sous nos climats, les poteaux d'incendie sont incongelables (frost-proof), grâce à leur dispositif de vidange (drain). À propos de ce dernier terme, il n'est pas superflu de rappeler que le mot français drain et ses dérivés ne sont usités qu'en agriculture, en médecine, en hydrographie, en géomorphologie et dans l'industrie du pétrole et du gaz. 
La bouche d'incendie (flush hydrant) est une \&prise d'eau normalisée, placée ainsi que ses organes de manouvre sous un couvercle ou une plaque de regard (lid), au niveau du sol ». Quant à la borne-fontaine (street fountain), c'est un appareil distributeur d'eau en forme de borne, disposé au-dessus d'un souillard (grating) fixé à la base du coffre. Il est muni d'un bec, appelé dégorgeoir (souvent à écoulement continu), et, accessoirement, d'une prise auxiliaire réservée en général à l'arrosage, utilisée exceptionnellement comme premier secours contre l'incendie. «Les bornes-fontaines sont utilisées dans les pares et les jardins publics ainsi que dans certaines localités où les habitants ne bénéficient pas encore de branchements particuliers. »

L'installation de chaque abonné est raccordée à la conduite d'eau de la ville (street main) au moyen d'un branchement d'eau (water service pipe) comportant un barrage de prise (city gate valve). Ce branchement alimente les canalisations du service journalier (domestic connections) et, éventuellement, le réseau d'eau d'incendie (fire protection piping system). Il est alors muni d'un compteur d'incendie (fire meter), de préférence à passage direct (free-flow meter). Dans les établissements industriels, ce réseau est approvisionné en tout ou en partie par le réseau d'eau industrielle (piping system for industrial process).

Le réseau d'eau d'incendie alimente, d'une part, à partir du poste de contrôle (valve room), les extincteurs automatiques à eau (automatic sprinkler system), notamment les rideaux d'eau (open head systems) — «barrages hydrauliques s'opposant au passage des flammes et au rayonnement, habituellement placés à proximité de portes ou de rideaux coupe-feu (fire doors and shutters) qu'ils ont pour but de refroidir $\gg$ - et, d'autre part, les canalisations d'incendie (stand-pipe system), sur lesquelles sont piqués les robinets armés (hose valves) de l'établissement et les prises de refoulement (hose connections) à l'usage des pompiers, placés dans les armoires d'incendie (hose cabinets), aux différents étages. La canalisation d'incendie est pourvue, en partie basse, d'un raccord pompier (fire department connection) permettant aux autopompes d'y refouler de l'eau en cas de sinistre.

L'alimentation des moyens de secours fixes contre l'incendie dont dispose l'établissement est soit directe (direct supply), soit en retour (gravity tank supply), lorsque des réservoirs sont disposés à la partie haute du bâtiment, soit par surpresseur (fire pump supply). Dans les deux derniers cas, la moto-pompe fixe (stationary building fire pump), installée dans une pomperie (pumping station), peut être alimentée par des canalisations en pression, ou encore aspirer l'eau à une plus ou moins grande profondeur (high, low suction lift), dans une citerne ou un puits. Son moteur (prime mover, driver) est tantôt un moteur électrique (motor), diesel, à essence ou à vapeur (engines), tantôt une turbine à vapeur (steam turbine). Son débit nominal (rated discharge capacity) doit être approprié aux risques (suitable to the hazards), notamment aux risques voisins (exposure hazards).

Bruno CoUture 